\title{
Wave front set for positive operators and for positive elements in non-commutative convolution algebras
}

\author{
by \\ JoACHIM TOFT (Växjö)
}

\begin{abstract}
Let $\mathrm{WF}_{*}$ be the wave front set with respect to $C^{\infty}$, quasi analyticity or analyticity, and let $K$ be the kernel of a positive operator from $C_{0}^{\infty}$ to $\mathscr{D}^{\prime}$. We prove that if $\xi \neq 0$ and $(x, x, \xi,-\xi) \notin \mathrm{WF}_{*}(K)$, then $(x, y, \xi,-\eta) \notin \mathrm{WF}_{*}(K)$ and $(y, x, \eta,-\xi) \notin$ $\mathrm{WF}_{*}(K)$ for any $y, \eta$. We apply this property to positive elements with respect to the weighted convolution
\end{abstract}

$$
u *_{B} \varphi(x)=\int u(x-y) \varphi(y) B(x, y) d y
$$

where $B \in C^{\infty}$ is appropriate, and prove that if $\left(u *_{B} \varphi, \varphi\right) \geq 0$ for every $\varphi \in C_{0}^{\infty}$ and $(0, \xi) \notin \mathrm{WF}_{*}(u)$, then $(x, \xi) \notin \mathrm{WF}_{*}(u)$ for any $x$.

0. Introduction. The aim of the paper is to discuss propagation of singularities in terms of different types of wave front sets for distribution kernels of positive operators, and for distributions which are positive with respect to some non-commutative convolution. For example we prove that kernels of positive operators in some sense are at most singular at the diagonal. As a consequence, a distribution which is positive with respect to some (non-commutative) convolution is at most singular at the origin. In particular we recover some results in $[4,12,17,18]$, where similar investigations were done for the usual convolution. We also recover some results in [15] where the twisted convolution is considered.

The positivity properties related to our investigations in part go back to [1], where Bochner proved that a function is positive definite if and only if it is the Fourier transform of a measure with finite mass. Recall that a (complex-valued) function $u$ on $\mathbb{R}^{n}$ is called positive definite if it is contin-

2000 Mathematics Subject Classification: Primary 35A18, 47B65, 35A21; Secondary $43 \mathrm{~A} 35,35 \mathrm{~S} 05$.

Key words and phrases: wave front set, non-commutative convolution, distribution kernel, positive operator. 
uous and for any finite sequences $\left\{x_{j}\right\} \subseteq \mathbb{R}^{n}$ and $\left\{c_{j}\right\} \subseteq \mathbb{C}$ we have

$$
\sum_{j, k} u\left(x_{j}-x_{k}\right) c_{j} \bar{c}_{k} \geq 0 .
$$

The latter condition on $u$ can be reformulated by means of the convolution $*$ between appropriate functions (or distributions) on $\mathbb{R}^{n}$. More precisely, it can be proved that $u$ is positive definite if and only if $u$ is continuous and $(u * \varphi, \varphi) \geq 0$ for every test function $\varphi$ on $\mathbb{R}^{n}$.

By taking this fact into account, Bochner's theorem can be formulated in the following way.

Assume that $u \in C\left(\mathbb{R}^{n}\right)$. Then $(u * \varphi, \varphi) \geq 0$ for every $\varphi \in C_{0}^{\infty}\left(\mathbb{R}^{n}\right)$ if and only if $\widehat{u}$ is a positive measure of finite mass, and then

$$
(2 \pi)^{-n / 2}\|\widehat{u}\|=\|u\|_{L^{\infty}}=u(0)(<\infty) .
$$

Here and in what follows we use the standard notation for the usual function and distribution spaces, and we let $(\cdot, \cdot)$ be the extension of the $L^{2}$ product from $C_{0}^{\infty}$ to $\mathscr{D}^{\prime} \times C_{0}^{\infty}$. (See e.g. [8].) Furthermore, we let the Fourier transform on $\mathscr{S}^{\prime}\left(\mathbb{R}^{n}\right)$ be chosen in such a way that it takes the form

$$
\mathscr{F} u(\xi)=\widehat{u}(\xi)=(2 \pi)^{-n / 2} \int u(x) e^{-i\langle x, \xi\rangle} d x
$$

when $u \in L^{1}\left(\mathbb{R}^{n}\right)$.

As a consequence of $(0.1)$, if $u$ is a positive definite function, then the total mass of $\widehat{u}$ only depends on the size of $u$ at the origin. It follows by straightforward approximations that the condition $u \in C\left(\mathbb{R}^{n}\right)$ in Bochner's theorem may be replaced by the weaker condition that $u \in \mathscr{D}^{\prime}\left(\mathbb{R}^{n}\right)$ is a continuous function near the origin (see e.g. [15] in the case of twisted convolution). The following "smooth Bochner theorem" is now a fairly simple consequence of Bochner's theorem (see e.g. $[4,12,17]$ or [18]).

Assume that $u \in \mathscr{D}^{\prime}\left(\mathbb{R}^{n}\right)$ is a positive definite function which is smooth near the origin. Then it is smooth everywhere.

In fact, if $u \in \mathscr{D}^{\prime}\left(\mathbb{R}^{n}\right)$ is smooth near the origin and $(u * \varphi, \varphi) \geq 0$ for every $\varphi \in C_{0}^{\infty}\left(\mathbb{R}^{n}\right)$, then the same is true with $u$ replaced by $(1-\Delta)^{N} u$. Hence Bochner's theorem shows that $\left(1+|\xi|^{2}\right)^{N} \widehat{u}(\xi)$ is a positive measure with finite mass. Since $N$ is arbitrary it is easily seen that the Fourier transform of $\widehat{u}$ must be smooth everywhere, i.e. $u(x)=\left(\mathscr{F}^{2} u\right)(-x)$ is smooth, which proves the assertion.

The smooth Bochner theorem can also be reformulated in terms of positive (semi-definite) operators. More precisely, assume that $T$ is a linear and continuous operator from $C_{0}^{\infty}\left(\mathbb{R}^{n}\right)$ to $\mathscr{D}^{\prime}\left(\mathbb{R}^{n}\right)$. Then $T$ is called positive (semi-definite) if $(T \varphi, \varphi) \geq 0$ for every $\varphi \in C_{0}^{\infty}\left(\mathbb{R}^{n}\right)$. By the kernel 
theorem of Schwartz there is a unique distribution $K \in \mathscr{D}^{\prime}\left(\mathbb{R}^{2 n}\right)$ (the kernel of $T)$ such that $(T \varphi, \psi)=(K, \psi \otimes \bar{\varphi})$ for every $\varphi, \psi \in C_{0}^{\infty}\left(\mathbb{R}^{n}\right)$. For convenience we let $\mathscr{D}_{0}^{\prime}\left(\mathbb{R}^{2 n}\right)$ be the set of all distribution kernels of positive operators from $C_{0}^{\infty}\left(\mathbb{R}^{n}\right)$ to $\mathscr{D}^{\prime}\left(\mathbb{R}^{n}\right)$. This means that $K \in \mathscr{D}_{\circ}^{\prime}\left(\mathbb{R}^{2 n}\right)$ if and only if $K \in \mathscr{D}^{\prime}\left(\mathbb{R}^{2 n}\right)$ satisfies

$$
(K, \varphi \otimes \bar{\varphi}) \geq 0 \quad \text { for every } \varphi \in C_{0}^{\infty}\left(\mathbb{R}^{n}\right) .
$$

Since the kernel of the operator $\varphi \mapsto u * \varphi$ is equal to $K(x, y)=$ $K_{u}(x, y)=u(x-y)$, it follows that $u$ is a positive definite function if and only if $K_{u}$ is continuous and belongs to $\mathscr{D}_{\circ}^{\prime}\left(\mathbb{R}^{2 n}\right)$. Evidently, the smoothness property for positive definite functions can be reformulated as:

Assume that $K_{u} \in \mathscr{D}_{\circ}^{\prime}\left(\mathbb{R}^{2 n}\right)$ is smooth along the diagonal $x=y$. Then it is smooth everywhere.

In Section 2 we extend the latter result in several directions. For example we prove that it holds for general $K \in \mathscr{D}_{\circ}^{\prime}\left(\mathbb{R}^{2 n}\right)$. These investigations are based on techniques which involve different types of wave front sets. Roughly speaking, the wave front set $\mathrm{WF}_{*}(u)$ of a distribution $u$ with respect to $C^{\infty}$ or $\mathscr{C}^{L}=C^{L}$ (see Sections 8.1 and 8.4 in [8], or Section 1 below for the definitions) gives information where the distribution is singular, i.e. where it fails to belong to $C^{\infty}$ or $\mathscr{C}^{L}$ respectively, as well as the directions of the propagating singularities.

In this context we prove in Section 2 the following:

Assume that $K \in \mathscr{D}_{\circ}^{\prime}\left(\mathbb{R}^{2 n}\right), \xi \in \mathbb{R}^{n} \backslash 0$ and $(x, x, \xi,-\xi) \notin \mathrm{WF}_{*}(K)$. Then $(x, y, \xi,-\eta) \notin \mathrm{WF}_{*}(K)$ and $(y, x, \eta,-\xi) \notin \mathrm{WF}_{*}(K)$ for any $y, \eta \in \mathbb{R}^{n}$. Consequently, if $K$ is smooth (resp. real-analytic) along the diagonal $x=y$, then it is smooth (resp. real-analytic) everywhere.

Furthermore, in Section 2 we prove that if $K \in \mathscr{S}^{\prime}\left(\mathbb{R}^{2 n}\right)$ and $K_{\mathscr{F}}(\xi, \eta)=$ $\widehat{K}(\xi,-\eta)$, then $K \in \mathscr{D}_{\circ}^{\prime}$ if and only if $K_{\mathscr{F}} \in \mathscr{D}_{\circ}^{\prime}$. Hence the latter wave front result gives similar wave front properties for the Fourier transform of elements in $\mathscr{D}_{0}^{\prime}$.

In Section 3 we apply these results to distributions which are positive with respect to some non-commutative convolution, where, in contrast to the usual convolution, a weight function is included in the definitions. More precisely, let $C^{*}\left(\mathbb{R}^{n}\right)$ be the set of all smooth and complex-valued functions on $\mathbb{R}^{n}$ which are non-zero everywhere, and assume that $B \in C^{*}\left(\mathbb{R}^{2 n}\right)$ and $u, \varphi \in C_{0}^{\infty}\left(\mathbb{R}^{n}\right)$. Then let

$$
u *_{B} \varphi(x)=\int u(x-y) \varphi(y) B(x, y) d y
$$

be the $B$-convolution of $u$ and $\varphi$. The definition of $*_{B}$ extends in the usual way to a continuous map from $\mathscr{D}^{\prime}\left(\mathbb{R}^{n}\right) \times C_{0}^{\infty}\left(\mathbb{R}^{n}\right)$ to $\mathscr{D}^{\prime}\left(\mathbb{R}^{n}\right) \cap C^{\infty}\left(\mathbb{R}^{n}\right)$. If 
in addition there exists a polynomial $P_{\alpha}$ on $\mathbb{R}^{2 n}$ such that

$$
\left|\partial^{\alpha} B\right|+|B|^{-1} \leq P_{\alpha}
$$

for every multi-index $\alpha$, then straightforward computations show that $*_{B}$ extends to a continuous map from $\mathscr{S}^{\prime}\left(\mathbb{R}^{n}\right) \times \mathscr{S}\left(\mathbb{R}^{n}\right)$ to $\mathscr{S}^{\prime}\left(\mathbb{R}^{n}\right) \cap C^{\infty}\left(\mathbb{R}^{n}\right)$.

We note that if $B=1$ everywhere, then $*_{B}$ coincides with the usual convolution. Assume instead that $\mathbb{R}^{2 n}$ is considered as the phase space with variables $X=(x, \xi) \in \mathbb{R}^{2 n}$ and $Y=(y, \eta) \in \mathbb{R}^{2 n}$, and let

$$
B(X, Y)=(2 / \pi)^{n / 2} e^{2 i \sigma(X, Y)}, \quad \text { where } \sigma(X, Y)=\langle y, \xi\rangle-\langle x, \eta\rangle
$$

is the symplectic form. Then $*_{B}$ agrees with the twisted convolution $*_{\sigma}$, which is important in the theory of pseudo-differential operators, since positivity and continuity properties in Weyl calculus can easily be reformulated in terms of positivity and continuity properties for the twisted convolution. (See $[7,14-16]$ or the end of Section 1.)

For an arbitrary $B \in C^{*}\left(\mathbb{R}^{2 n}\right)$, we are especially concerned with the set $\mathscr{D}_{B,+}^{\prime}\left(\mathbb{R}^{n}\right)$, consisting of all $u \in \mathscr{D}^{\prime}\left(\mathbb{R}^{n}\right)$ such that $\left(u *_{B} \varphi, \varphi\right) \geq 0$ for every $\varphi \in C_{0}^{\infty}\left(\mathbb{R}^{n}\right)$. In particular, $\mathscr{D}_{1,+}^{\prime}$ is the set of positive definite distributions.

Since the distribution kernel of the operator $\varphi \mapsto u *_{B} \varphi$ is equal to $K_{B, u}(x, y) \equiv u(x-y) B(x, y)$, it follows that $u \in \mathscr{D}_{B,+}^{\prime}\left(\mathbb{R}^{n}\right)$ if and only if $K_{B, u} \in \mathscr{D}_{\circ}^{\prime}\left(\mathbb{R}^{2 n}\right)$. In particular, any smoothness or wave front property on $\mathscr{D}_{\circ}^{\prime}$ carries over to $\mathscr{D}_{B,+}^{\prime}$, and in Section 3 we apply the results in Section 2 to prove the following:

Assume that $B \in C^{*}\left(\mathbb{R}^{2 n}\right)$ is "appropriate", $u \in \mathscr{D}_{B,+}^{\prime}\left(\mathbb{R}^{n}\right)$ and $(0, \xi) \notin$ $\mathrm{WF}_{*}(u)$. Then $(x, \xi) \notin \mathrm{WF}_{*}(u)$. Consequently, if $u$ is smooth (resp. realanalytic) near the origin, then it is smooth (resp. real-analytic) everywhere.

Here the condition that $B$ should be "appropriate" means that some extra assumptions are included, which depend on the wave front sets under consideration.

In the last section we make some additional remarks on $\mathscr{S}_{+}^{\prime}$, the set of distributions which are positive with respect to the twisted convolution. In [16] it is proved that if $u \in \mathscr{S}_{+}^{\prime}$ and $(0, Y) \notin \mathrm{WF}(u)$ for some $Y \in \mathbb{R}^{2 n} \backslash 0$, then $u \in \mathscr{S}^{\prime},(X, Y) \notin \mathrm{WF}(u)$ and $(X, Y) \notin \mathrm{WF}\left(\mathscr{F}_{\sigma} u\right)$ for all $X \in \mathbb{R}^{2 n}$. Here WF denotes the wave front set with respect to $C^{\infty}$ and $\mathscr{F}_{\sigma}$ denotes the symplectic Fourier transform (see Section 1 for precise definitions). In Section 4 we extend the latter property and prove that it also holds for other types of wave front set.

Finally, we remark that there are other ways to define wave front sets of different types (see e.g. $[2,9]$ ). 
1. Preliminaries. In this section we recall the definition of wave front set and present some facts which, in part, go back to [8]. We also recall some facts on the twisted convolution.

We start by recalling the definition of the wave front set with respect to $C^{\infty}\left(\mathbb{R}^{n}\right)$. Assume that $u \in \mathscr{S}^{\prime}\left(\mathbb{R}^{n}\right)$. Then the cone $\Theta(u)$ consists of all $\xi_{0} \in \mathbb{R}^{n} \backslash 0$ such that for some open conic neighbourhood $\Gamma$ of $\xi_{0}$ and each integer $N \geq 1$, there is a constant $C_{N}$ such that

$$
|\widehat{u}(\xi)| \leq C_{N}\langle\xi\rangle^{-N}, \quad \xi \in \Gamma
$$

Here and in what follows we let $\langle\xi\rangle=\left(1+|\xi|^{2}\right)^{1 / 2}$. We also let $\Sigma(u)=$ $\left\{\xi \in \mathbb{R}^{n} \backslash 0 ; \xi \notin \Theta(u)\right\}$. Furthermore, for each $x \in \mathbb{R}^{n}$ and $u \in \mathscr{D}^{\prime}\left(\mathbb{R}^{n}\right)$ we set

$$
\Sigma_{x}(u) \equiv \bigcap \Sigma(\chi u)
$$

where the intersection is taken over all $\chi \in C_{0}^{\infty}\left(\mathbb{R}^{n}\right)$ such that $\chi(x) \neq 0$.

Definition 1.1. Assume that $u \in \mathscr{D}^{\prime}\left(\mathbb{R}^{n}\right)$. Then the wave front set of $u$ (with respect to $C^{\infty}$ ) is the set

$$
\mathrm{WF}(u) \equiv\left\{(x, \xi) \in \mathbb{R}^{n} \times\left(\mathbb{R}^{n} \backslash 0\right) ; \xi \in \Sigma_{x}(u)\right\} .
$$

The following lemma might be helpful when computing the wave front set. We refer to (8.1.5) in [8] and its motivation for the proof.

Lemma 1.2. Assume that $\chi_{N} \in C_{0}^{\infty}\left(\mathbb{R}^{n}\right), N \geq 1$, are such that $\chi_{N}\left(x_{0}\right) \neq 0$ for every $N \geq 1$ and $\operatorname{supp} \chi_{N} \searrow\left\{x_{0}\right\}$ (i.e. the supports of $\chi_{N}$ decrease with $N$ and $\left.\bigcap_{N} \operatorname{supp} \chi_{N}=\left\{x_{0}\right\}\right)$. Then $\Sigma\left(\chi_{N} u\right) \rightarrow \Sigma_{x}(u)$.

Next we recall other types of wave front sets which are more related to propagation of singularities in the context of analyticity and quasi analyticity. From now on we assume that $L=\left\{L_{k}\right\}_{k \geq 0}$ is an increasing sequence of positive real numbers such that

$$
L_{0}=1, \quad L_{k} \geq k, \quad L_{k+1} \leq C L_{k}
$$

for some constant $C$ independent of $k$. If $\Omega \subseteq \mathbb{R}^{n}$ is open, then $\mathscr{C}^{L}(\Omega)$ is the set of all smooth functions $f$ on $\mathbb{R}^{n}$ such that for every compact set $K \subseteq \Omega$, there is a constant $C_{K}$ such that

$$
\left|D^{\alpha} f(x)\right| \leq C_{K}\left(C_{K} L_{|\alpha|}\right)^{|\alpha|}
$$

for all multi-indices $\alpha$ and $x \in K$.

We note that if $L_{k}=k$ when $k \geq 1$, then $\mathscr{C}^{L}\left(\mathbb{R}^{n}\right)$ coincides with $\mathcal{A}\left(\mathbb{R}^{n}\right)$, the set of real-analytic functions on $\mathbb{R}^{n}$. More generally, if it is only assumed that

$$
\sum_{k=1}^{\infty} \frac{1}{L_{k}}=\infty
$$


then $\mathscr{C}^{L}\left(\mathbb{R}^{n}\right)$ is contained in the set of quasi analytic functions on $\mathbb{R}^{n}$. (See [8].)

Next we recall the definition of the wave front set with respect to $\mathscr{C}^{L}$. If (1.3) is violated (i.e. $\mathscr{C}^{L}$ contains functions which are not quasi analytic), then there exist non-trivial functions in $\mathscr{C}^{L} \cap C_{0}^{\infty}$. In this case, the wave front set with respect to $\mathscr{C}^{L}$ can be defined similarly to the wave front set with respect to $C^{\infty}$. In order to obtain a definition of wave front sets which fits all situations we may as in [8] proceed as follows. Assume that $u \in \mathscr{D}^{\prime}\left(\mathbb{R}^{n}\right)$ and $x \in \mathbb{R}^{n}$. Let $\Theta_{x}^{L}(u)$ be the cone of all $\xi_{0} \in \mathbb{R}^{n} \backslash 0$ such that for some open neighbourhood $U$ of $x$, conical neighbourhood $\Gamma$ of $\xi_{0}$, constant $C$ and bounded sequence $\left\{u_{N}\right\}$ in $\mathscr{E}^{\prime}\left(\mathbb{R}^{n}\right)$ such that $u_{N}=u$ on $U$, we have

$$
\left|\mathscr{F}\left(u_{N}\right)(\xi)\right| \leq C\left(C L_{N} /|\xi|\right)^{N}, \quad \xi \in \Gamma .
$$

We also set $\Sigma_{x}^{L}(u)=\left\{\xi \in \mathbb{R}^{n} \backslash 0 ; \xi \notin \Theta_{x}^{L}(u)\right\}$. Then $\Sigma_{x}^{L}(u)=\emptyset$ if and only if $u \in \mathscr{C}^{L}$ near $x$ (see e.g. [8]).

Definition 1.3. Assume that $u \in \mathscr{D}^{\prime}\left(\mathbb{R}^{n}\right)$. Then the wave front set with respect to $\mathscr{C}^{L}\left(\mathbb{R}^{n}\right)$ of $u$ is the set

$$
\mathrm{WF}_{L}(u)=\left\{(x, \xi) \in \mathbb{R}^{n} \times\left(\mathbb{R}^{n} \backslash 0\right) ; \xi \in \Sigma_{x}^{L}(u)\right\} .
$$

If $L_{k}=k$ for all $k \geq 1$, then $\mathrm{WF}_{L}(u)$ is called the analytic wave front set of $u$ and is denoted by $\mathrm{WF}_{\mathcal{A}}(u)$.

We recall that for every $u \in \mathscr{D}^{\prime}\left(\mathbb{R}^{n}\right)$ and all permitted sequences $L$,

$$
\mathrm{WF}(u) \subseteq \mathrm{WF}_{L}(u) \subseteq \mathrm{WF}_{\mathcal{A}}(u)
$$

The next lemma may be useful when computing the wave front set for a distribution with respect to $\mathscr{C}^{L}$. The proof is omitted, since the lemma is essentially a restatement of Lemma 8.4.4 in [8]. Here and in what follows we let $\Lambda^{L}(\Omega)$ for $\Omega \subseteq \mathbb{R}^{n}$ be the set of all sequences $\left\{\chi_{N}\right\}_{N \geq 1}$ in $C^{\infty}\left(\mathbb{R}^{n}\right)$ such that $\operatorname{supp} \chi_{N} \subseteq \Omega$ for every $N \geq 1$, and for every multi-index $\alpha$ there is a constant $C_{\alpha}$ such that

$$
\left|D^{\alpha+\beta} \chi_{N}\right| \leq C_{\alpha}\left(C_{\alpha} L_{N}\right)^{|\beta|}, \quad|\beta| \leq N .
$$

Lemma 1.4. Assume that $u \in \mathscr{D}^{\prime}\left(\mathbb{R}^{n}\right), K \subseteq \mathbb{R}^{n}$ is compact, and $F$ is a closed cone in $\mathbb{R}^{n} \backslash 0$ such that $\mathrm{WF}_{L}(u) \cap K \times F=\emptyset$. Also assume that $\left\{\chi_{N}\right\}_{N \geq 1} \in \Lambda^{L}(K)$. Then $\chi_{N} u$ is bounded in $\mathscr{E}^{M}$ if $M$ is the order of $u$ in a neighbourhood of $K$, and

$$
\left|\mathscr{F}\left(\chi_{N} u\right)(\xi)\right| \leq C\left(C L_{N} /|\xi|\right)^{N}, \quad \xi \in F
$$

for some constant $C$ independent of $N \geq 1$.

We also need the following refinement of Lemma 1.4. Again the proof is omitted since the result follows from the same arguments as in the proof of Lemma 8.4.4 in [8]. 
Lemma 1.5. Assume that $K \subseteq \mathbb{R}^{n}$ is compact and $F \subseteq \Gamma$ are cones such that $F$ is closed and $\Gamma$ is open in $\mathbb{R}^{n} \backslash\{0\}$. Also assume that $\left\{\chi_{N}\right\}_{N \geq 1} \in$ $\Lambda^{L}(K)$ and $\left\{u_{N}\right\}_{N \geq 1}$ is a bounded subset of $\mathscr{E}^{\prime}(K)$ such that (1.4) holds for some constant $C$ independent of $N \geq 1$. Then

$$
\left|\mathscr{F}\left(\chi_{N} u_{N}\right)(\xi)\right| \leq C\left(C L_{N} /|\xi|\right)^{N}, \quad \xi \in F,
$$

for some constant $C$ independent of $N \geq 1$.

We also need the following converse of Lemmas 1.4 and 1.5.

Lemma 1.6. Assume that $K \subseteq \mathbb{R}^{n}$ is compact, $U \subseteq K$ is an open neighbourhood of $x_{0} \in K$, and $\xi_{0} \in \Gamma$, where $\Gamma$ is an open cone in $\mathbb{R}^{n} \backslash\{0\}$. Also assume that $\left\{\chi_{N}\right\}_{N \geq 1} \in \Lambda^{L}\left(\mathbb{R}^{n}\right)$ with $\left|\chi_{N}(x)\right| \geq c$ when $x \in U$ for some constant $c>0$ independent of $N$. If $u \in \mathscr{D}^{\prime}\left(\mathbb{R}^{n}\right)$ and (1.4) holds with $u_{N}=\chi_{N} u$, then $\left(x_{0}, \xi_{0}\right) \notin \mathrm{WF}_{L}(u)$.

In the proof as well as later on we let $B_{r}(a)$ denote the open ball with centre at $a$ and radius $r$.

Proof. Choose $r>0$ such that $B_{3 r}\left(x_{0}\right) \subseteq U$. By the $\mathscr{C}^{L}$-calculus of [8] there exists a sequence $\left\{\psi_{N}\right\}_{N \geq 1} \in \Lambda^{L}\left(\complement\left(B_{r}\left(x_{0}\right)\right)\right)$ such that

$$
0 \leq \psi_{N} \leq 1 \quad \text { and } \quad \psi_{N}(x)=1 \quad \text { when } x \notin B_{3 r}\left(x_{0}\right) .
$$

(See e.g. the end of the proof of Lemma 8.4.4 in [8].) Then

$$
\varphi_{N}=\bar{\chi}_{N} /\left(\psi_{N}+\left|\chi_{N}\right|^{2}\right) \in C_{0}^{\infty}(K)
$$

satisfies (1.5) with $\chi_{N}$ replaced by $\varphi_{N}$, and $C_{\alpha}$ replaced by larger constants if necessary.

Now let $v_{N}=\varphi_{N} \chi_{N} u=\varphi_{N} u_{N}$. Then $\left\{v_{N}\right\}$ is a bounded set in $\mathscr{E}^{\prime}(K)$. If $F$ is a closed conic neighbourhood of $\xi_{0}$ such that $F \subseteq \Gamma$, then Lemma 1.5 shows that

$$
\left|\widehat{v}_{N}(\xi)\right| \leq C\left(C L_{N} /|\xi|\right)^{N}, \quad \xi \in F .
$$

Since $v_{N}=u$ in $B_{r}\left(x_{0}\right)$, the last inequality means that $\left(x_{0}, \xi_{0}\right) \notin \mathrm{WF}_{L}(u)$.

REMARK 1.7. We note that for any sequence $L=\left\{L_{0}, L_{1}, \ldots\right\}$ which satisfies the required conditions, we can always find a sequence $\left\{\chi_{N}\right\}_{N \geq 0}$ such that the assumptions in Lemma 1.6 are fulfilled. (Cf. Sections 1.4 and 8.4 in [8].)

We finish the section by giving some remarks on twisted convolution. Recall that if $u, \psi \in L^{1}\left(\mathbb{R}^{2 n}\right)$, then the twisted convolution of $u$ and $\psi$ is defined by the formula

$$
\left(u *_{\sigma} \psi\right)(X)=(2 / \pi)^{n / 2} \int u(X-Y) \psi(Y) e^{2 i \sigma(X, Y)} d Y
$$

Here $\mathbb{R}^{2 n}$ is considered as the phase space with variables $X=(x, \xi) \in \mathbb{R}^{2 n}$ and $Y=(y, \eta) \in \mathbb{R}^{2 n}$, and $\sigma(X, Y)=\langle y, \xi\rangle-\langle x, \eta\rangle$ is the (standard) symplectic form on $\mathbb{R}^{2 n}$. 
The twisted convolution is briefly investigated in [7, 14-16], where connections between twisted convolution and Weyl calculus of pseudo-differential operators, concerning positivity and continuity, are presented. Recall that if $u \in \mathscr{S}\left(\mathbb{R}^{2 n}\right)$, then the Weyl operator for $u$ is defined by

$$
u^{w}(x, D) f(x)=(2 \pi)^{-n} \iint u((x+y) / 2, \xi) f(y) e^{i\langle x-y, \xi\rangle} d y d \xi
$$

when $f \in \mathscr{S}\left(\mathbb{R}^{n}\right)$. It follows that $u^{w}(x, D)$ is continuous on $\mathscr{S}\left(\mathbb{R}^{n}\right)$. Furthermore, the definition of $u^{w}(x, D)$ extends to any $u \in \mathscr{S}^{\prime}\left(\mathbb{R}^{2 n}\right)$, and then $u^{w}(x, D)$ is continuous from $\mathscr{S}\left(\mathbb{R}^{n}\right)$ to $\mathscr{S}^{\prime}\left(\mathbb{R}^{n}\right)$. (See [8].)

In Proposition 1.8 below we point out some important links between twisted convolution and Weyl calculus. Here and in what follows, $\mathscr{F}_{\sigma}$ denotes the symplectic Fourier transform, defined by the formula

$$
\mathscr{F}_{\sigma} u(X)=\widehat{u}(X) \equiv \pi^{-n} \int u(Y) e^{2 i \sigma(X, Y)} d Y
$$

when $u \in L^{1}\left(\mathbb{R}^{2 n}\right)$. Then $\mathscr{F}_{\sigma}$ is continuous on $\mathscr{S}\left(\mathbb{R}^{2 n}\right)$ and extends in the usual way to a continuous map on $\mathscr{S}^{\prime}\left(\mathbb{R}^{2 n}\right)$ and on $L^{2}\left(\mathbb{R}^{2 n}\right)$. Furthermore, $\mathscr{F}_{\sigma}^{2}$ is the identity operator. We also let $\check{u}(x)=u(-x)$ and $\widetilde{u}(x)=\overline{u(-x)}$ when $u \in \mathscr{D}^{\prime}\left(\mathbb{R}^{n}\right)$.

Proposition 1.8. Assume that $u \in \mathscr{S}^{\prime}\left(\mathbb{R}^{2 n}\right)$ and $v, w \in \mathscr{S}\left(\mathbb{R}^{2 n}\right)$, and that (0.4) holds. Then the following hold:

(1) $\left(u *_{\sigma} v, w\right)=\left(u, w *_{\sigma} \widetilde{v}\right)$;

(2) $\left(u *_{\sigma} v\right) *_{\sigma} w=u *_{\sigma}\left(v *_{\sigma} w\right)$;

(3) $\mathscr{F}_{\sigma}\left(u *_{\sigma} v\right)=\left(\mathscr{F}_{\sigma} u\right) *_{\sigma} v=\check{u} *_{\sigma}\left(\mathscr{F}_{\sigma} v\right)$;

(4) $u^{w}(x, D) \geq 0$ if and only if $\mathscr{F}_{\sigma} u \in \mathscr{S}_{+}^{\prime}\left(\mathbb{R}^{2 n}\right)$;

(5) $u^{w}(x, D) v^{w}(x, D)=(2 \pi)^{-n / 2}\left(u *_{\sigma}\left(\mathscr{F}_{\sigma} v\right)\right)^{w}(x, D)$.

2. Wave front set of kernels of positive operators. In this section we discuss the properties of the wave front set of kernels of positive operators from $C_{0}^{\infty}$ to $\mathscr{D}^{\prime}$. Recall from the introduction that $K$ is the Schwartz kernel of a positive operator if and only if $(0.2)$ holds. Also recall that $\mathscr{D}_{0}^{\prime}\left(\mathbb{R}^{2 n}\right)$ is the set of all kernels $K \in \mathscr{D}^{\prime}\left(\mathbb{R}^{2 n}\right)$ of positive operators.

We start by discussing invariance properties of $\mathscr{D}_{\circ}^{\prime}\left(\mathbb{R}^{2 n}\right)$ under multiplication with elements in $\mathscr{D}_{1,+}^{\prime}$ (cf. the introduction). Here and in what follows it is convenient to set $f_{\tau}(x, y)=f(x-y)$ when $f \in \mathscr{D}^{\prime}\left(\mathbb{R}^{n}\right)$.

Proposition 2.1. Assume that $K \in \mathscr{D}_{\circ}^{\prime}\left(\mathbb{R}^{2 n}\right)$ and $f \in \mathscr{D}_{1,+}^{\prime}\left(\mathbb{R}^{n}\right) \cap$ $C^{\infty}\left(\mathbb{R}^{n}\right)$. Then $f_{\tau} K \in \mathscr{D}_{\circ}^{\prime}\left(\mathbb{R}^{2 n}\right)$.

Remark 2.2. Assume that $K \in \mathscr{D}_{\circ}^{\prime}\left(\mathbb{R}^{2 n}\right)$ and $\xi \in \mathbb{R}^{n}$. Then Proposition 2.1 shows that if $K_{1}(x, y)=e^{i\langle x-y, \xi\rangle} K(x, y)$, then $K_{1} \in \mathscr{D}_{\circ}^{\prime}\left(\mathbb{R}^{2 n}\right)$, since $e^{i\langle\cdot, \xi\rangle} \in \mathscr{D}_{1,+}^{\prime} \cap C^{\infty}$. 
Proof of Proposition 2.1. Let $K_{1}=f_{\tau} K$. We first prove the assertion when $f=h_{\xi} \equiv e^{i\langle\cdot, \xi\rangle}\left(\in \mathscr{D}_{1,+}^{\prime} \cap C^{\infty}\right)$. Let $\varphi \in C_{0}^{\infty}\left(\mathbb{R}^{n}\right)$. Then straightforward computations yield

$$
\left(K_{1}, \varphi \otimes \bar{\varphi}\right)=\left(K,\left(e^{-i\langle\cdot, \xi\rangle} \varphi\right) \otimes\left(\overline{e^{-i\langle\cdot, \xi\rangle} \varphi}\right)\right) \geq 0,
$$

and the assertion follows.

Next assume that $f \in \mathscr{D}_{1,+}^{\prime} \cap C^{\infty}$ and $\mu=\widehat{f}$ has compact support. Since $\mu$ is a positive measure, the first part of the proof implies

$$
\begin{aligned}
0 & \leq(2 \pi)^{-n / 2} \int\left(\left(h_{\xi}\right)_{\tau} K, \varphi \otimes \bar{\varphi}\right) d \mu(\xi) \\
& =\left((2 \pi)^{-n / 2}\left(\int h_{\xi} d \mu(\xi)\right)_{\tau} K, \varphi \otimes \bar{\varphi}\right)=\left(f_{\tau} K, \varphi \otimes \bar{\varphi}\right),
\end{aligned}
$$

and the assertion follows in this case.

Finally, let $f \in \mathscr{D}_{1,+}^{\prime} \cap C^{\infty}$ be arbitrary. For every $\varepsilon>0$, let $f_{\varepsilon} \in$ $\mathscr{D}_{1,+}^{\prime} \cap C^{\infty}$ be defined by the formula $\widehat{f_{\varepsilon}}=\widehat{f} \chi(\varepsilon \cdot)$, where $\chi \in C_{0}^{\infty}\left(\mathbb{R}^{n}\right)$ is fixed and satisfies $0 \leq \chi \leq 1$ and $\chi(0)=1$. Since $\widehat{f}$ is a positive measure with bounded mass, it follows that the same is true for $f_{\varepsilon}$. Furthermore, $\widehat{f}_{\varepsilon}$ has compact support and $f_{\varepsilon} \rightarrow f$ in $C^{\infty}$ as $\varepsilon \rightarrow 0$. Hence by the first part of the proof,

$$
\left(f_{\tau} K, \varphi \otimes \bar{\varphi}\right)=\lim _{\varepsilon \rightarrow 0}\left(\left(f_{\varepsilon}\right)_{\tau} K, \varphi \otimes \bar{\varphi}\right) \geq 0 \quad \text { when } \varphi \in C_{0}^{\infty}\left(\mathbb{R}^{n}\right) .
$$

The next result concerns the smallest wave front set of elements in $\mathscr{D}_{0}^{\prime}$, i.e. the one with respect to $C^{\infty}\left(\mathbb{R}^{n}\right)$.

Theorem 2.3. Assume that $K \in \mathscr{D}_{\circ}^{\prime}\left(\mathbb{R}^{2 n}\right)$ and $x, y, \eta \in \mathbb{R}^{n}$. If $\xi \in \mathbb{R}^{n} \backslash 0$ and $(x, x, \xi,-\xi) \notin \mathrm{WF}(K)$, then $(x, y, \xi,-\eta) \notin \mathrm{WF}(K)$ and $(y, x, \eta,-\xi) \notin$ $\mathrm{WF}(K)$.

Proof. First note that the positivity of $K$ implies that

$$
\left(\psi_{1}, \psi_{2}\right) \mapsto\left(K, \psi_{2} \otimes \overline{\psi_{1}}\right)
$$

is a semi-scalar product on $C_{0}^{\infty}$. Hence Cauchy's inequality holds for this product, i.e.

$$
|(K, \varphi \otimes \bar{\psi})|^{2} \leq(K, \varphi \otimes \bar{\varphi})(K, \psi \otimes \bar{\psi})
$$

for all $\varphi, \psi \in C_{0}^{\infty}\left(\mathbb{R}^{n}\right)$.

Let now $\varphi \in C_{0}^{\infty}\left(\mathbb{R}^{n}\right)$ be such that $\varphi(0) \neq 0$, and let $\varphi_{x}=\varphi(\cdot-x)$ for $x \in \mathbb{R}^{n}$. Choose $x_{0}$ and $\xi_{0} \neq 0$ with $\left(x_{0}, x_{0}, \xi_{0},-\xi_{0}\right) \notin \mathrm{WF}(K)$, and let $y_{0} \in \mathbb{R}^{n}$,

$$
\chi=\bar{\varphi}_{x_{0}} \otimes \varphi_{y_{0}}, \quad \chi_{1}=\bar{\varphi}_{x_{0}} \otimes \varphi_{x_{0}}, \quad \chi_{2}=\bar{\varphi}_{y_{0}} \otimes \varphi_{y_{0}}
$$


By the Cauchy-Schwarz inequality we get

$$
\begin{aligned}
\mid \mathscr{F}(\chi K) & \left.(\xi,-\eta)\right|^{2}=\left|\left(K,\left(\varphi_{x} e^{i\langle\cdot, \xi\rangle}\right) \otimes \overline{\left(\varphi_{y} e^{i\langle\cdot, \eta\rangle}\right)}\right)\right|^{2} \\
& \leq\left(K,\left(\varphi_{x} e^{i\langle\cdot, \xi\rangle}\right) \otimes \overline{\left(\varphi_{x} e^{i\langle\cdot, \xi\rangle}\right)}\right)\left(K,\left(\varphi_{y} e^{i\langle\cdot, \eta\rangle}\right) \otimes \overline{\left(\varphi_{y} e^{i\langle\cdot, \eta\rangle}\right)}\right) \\
& =\mathscr{F}\left(\chi_{1} K\right)(\xi,-\xi) \mathscr{F}\left(\chi_{2} K\right)(\eta,-\eta) .
\end{aligned}
$$

By choosing $\varphi$ with support small enough, it follows from the assumptions that for some conical neighbourhood $\Gamma$ of $\xi_{0}$ we have

$$
\left|\mathscr{F}\left(\chi_{1} K\right)\left(\xi_{1},-\xi_{2}\right)\right| \leq C_{N}\left\langle\left(\xi_{1}, \xi_{2}\right)\right\rangle^{-N}, \quad \xi_{1}, \xi_{2} \in \Gamma, N=1,2, \ldots
$$

Furthermore, since $\chi_{2} K$ has compact support,

$$
\left|\mathscr{F}\left(\chi_{2} K\right)\left(\eta_{1}, \eta_{2}\right)\right| \leq C\left\langle\left(\eta_{1}, \eta_{2}\right)\right\rangle^{M}, \quad \eta_{1}, \eta_{2} \in \mathbb{R}^{n},
$$

for some $M<\infty$.

Next let $\eta_{0} \in \mathbb{R}^{n}$ be arbitrary, choose $r>0$ such that $B_{2 r}\left(\xi_{0}\right) \subseteq \Gamma$, and let $\Gamma_{0}$ be the smallest open cone in $\mathbb{R}^{2 n} \backslash 0$ which contains the ball $B_{r}\left(\xi_{0},-\eta_{0}\right)$ in $\mathbb{R}^{2 n}$. Then there is a constant $c>0$ such that $|\xi| \geq c$ when $(\xi, \eta) \in B_{r}\left(\xi_{0},-\eta_{0}\right)$. This gives

$$
C|(\xi,-\eta)| \leq|\xi| \leq|(\xi,-\eta)|, \quad C|\eta| \leq|\xi|, \quad(\xi,-\eta) \in \Gamma_{0},
$$

for some constant $C>0$.

Hence a combination of (2.3)-(2.6) gives

$$
\begin{aligned}
& |\mathscr{F}(\chi K)(\xi,-\eta)|^{2} \leq \mathscr{F}\left(\chi_{1} K\right)(\xi,-\xi) \mathscr{F}\left(\chi_{2} K\right)(\eta,-\eta) \\
& \leq C C_{N+M}\langle\xi\rangle^{-(N+M)}\langle\eta\rangle^{M} \leq C^{\prime} C_{N}\langle\xi\rangle^{-N} \leq C^{\prime \prime} C_{N}\langle(\xi, \eta)\rangle^{-N}
\end{aligned}
$$

when $(\xi,-\eta) \in \Gamma_{0}$. This proves that $\left(\xi_{0},-\eta_{0}\right) \notin \Sigma(\chi K)$. If $\chi$ is replaced by $\chi_{0}=\bar{\varphi}_{y_{0}} \otimes \varphi_{x_{0}}$, then similar calculations yield $\left(\eta_{0},-\xi_{0}\right) \notin \Sigma(\chi K)$. This proves that $\left(x_{0}, y_{0}, \xi_{0},-\eta_{0}\right) \notin \mathrm{WF}(K)$ and $\left(y_{0}, x_{0}, \eta_{0},-\xi_{0}\right) \notin \mathrm{WF}(K)$, and the result follows.

As an immediate consequence of Theorem 2.3 we have the following.

Corollary 2.4. Assume that $K \in \mathscr{D}_{\circ}^{\prime}\left(\mathbb{R}^{2 n}\right) \cap C^{\infty}(U)$, where $U \subseteq \mathbb{R}^{2 n}$ is an open neighbourhood of the diagonal $x=y$. Then $K \in C^{\infty}\left(\mathbb{R}^{n}\right)$.

Next we discuss wave front properties with respect to $\mathscr{C}^{L}$. The following result corresponds to Theorem 2.3.

Theorem 2.5. Assume that $K \in \mathscr{D}_{\circ}^{\prime}\left(\mathbb{R}^{2 n}\right)$ and $x, y, \eta \in \mathbb{R}^{n}$. If $\xi \in \mathbb{R}^{n} \backslash 0$ and $(x, x, \xi,-\xi) \notin \mathrm{WF}_{*}(K)$, then $(x, y, \xi,-\eta) \notin \mathrm{WF}_{*}(K)$ and $(y, x, \eta,-\xi) \notin$ $\mathrm{WF}_{*}(K)$, where $\mathrm{WF}_{*}$ stands for $\mathrm{WF}_{L}$ or $\mathrm{WF}_{\mathcal{A}}$.

For the proof we need the following lemma.

Lemma 2.6. Assume that $U_{1}, U_{2} \subseteq \mathbb{R}^{n},\left\{\varphi_{N}\right\} \in \Lambda^{L}\left(U_{1}\right)$ and $\left\{\psi_{N}\right\} \in$ $\Lambda^{L}\left(U_{2}\right)$. Then $\left\{\varphi_{N} \otimes \psi_{N}\right\} \in \Lambda^{L}\left(U_{1} \times \bar{U}_{2}\right)$. 
Proof. This follows immediately from Proposition 8.4.1 in [8] and its proof.

Proof of Theorem 2.5. It suffices to prove the assertion for $\mathrm{WF}_{L}$. Choose $x_{0}, \xi_{0} \in \mathbb{R}^{n}$ with $\xi_{0} \neq 0$ and $\left(x_{0}, x_{0}, \xi_{0},-\xi_{0}\right) \notin \mathrm{WF}_{L}(K)$, and set $U_{x, y}=$ $(x, y)+U$, when $U \subseteq \mathbb{R}^{2 n}$ and $x, y \in \mathbb{R}^{n}$. From the assumptions it follows that there is a neighbourhood $U \subseteq \mathbb{R}^{2 n}$ of the origin, an open conical neighbourhood $\Gamma$ of $\xi_{0}$, a constant $C>0$ and a bounded sequence $\left\{K_{N}\right\}_{N \geq 0}$ in $\mathscr{E}^{\prime}$ such that $K_{N}=K$ on $U_{x_{0}, x_{0}}$ and

$$
\left|\mathscr{F}\left(K_{N}\right)(\xi,-\eta)\right| \leq C\left(C L_{N} /|(\xi, \eta)|\right)^{N}, \quad \xi, \eta \in \Gamma .
$$

Next let $y_{0} \in \mathbb{R}^{n}$ be arbitrary, and choose $r_{1}>0$ such that $B_{2 r_{1}}(0) \subseteq U$. Also let $\varphi_{N} \in C_{0}^{\infty}\left(\mathbb{R}^{n}\right)$ be such that $\left\{\varphi_{N}\right\} \in \Lambda^{L}\left(B_{2 r}(0)\right)$ and $\varphi_{N}(x)=1$ when $x \in B_{r_{1}}(0)$. Then it follows from Lemma 2.6 that if

$$
\chi_{N}=\overline{\varphi_{N}\left(\cdot-x_{0}\right)} \otimes \varphi_{N}\left(\cdot-y_{0}\right),
$$

then $\left\{\chi_{N}\right\} \in \Lambda^{L}\left(B_{2 r}\left(x_{0}, y_{0}\right)\right)$, and for some $r_{0}>0$,

$$
\chi_{N}(x, y)=1 \quad \text { when }(x, y) \in B_{r_{0}}\left(x_{0}, y_{0}\right) .
$$

By (2.3) we get

$$
\left|\mathscr{F}\left(\chi_{N} K\right)(\xi,-\eta)\right|^{2} \leq \mathscr{F}\left(\chi_{1, N} K\right)(\xi,-\xi) \mathscr{F}\left(\chi_{2, N} K\right)(\eta,-\eta) \mid
$$

where

$$
\chi_{1, N}=\overline{\varphi_{N}\left(\cdot-x_{0}\right)} \otimes \varphi_{N}\left(\cdot-x_{0}\right), \quad \chi_{2, N}=\overline{\varphi_{N}\left(\cdot-y_{0}\right)} \otimes \varphi_{N}\left(\cdot-y_{0}\right)
$$

Since $\left\{\chi_{1, N}\right\} \in \Lambda^{L}\left(B_{2 r}\left(x_{0}, x_{0}\right)\right)$, Lemma 1.4 implies that (2.7) holds for $K_{1, N}=\chi_{1, N} K$, with $\Gamma$ replaced by a smaller conical neighbourhood of $\xi_{0}$ if necessary.

Next let $\eta_{0}, r$ and $\Gamma_{0}$ be as in the proof of Theorem 2.3. Since $\chi_{2, N} K$ is a bounded set in $\mathscr{E}^{\prime}\left(U_{\left(y_{0}, y_{0}\right)}\right)$, it follows that (2.5) holds when $\chi_{2}=\chi_{2, N}$, for some constants $C$ and $M$ independent of $N$. By repeating the arguments in the proof of Theorem 2.3, a combination of $(2.5)-(2.9)$ now gives

$$
\left|\mathscr{F}\left(\chi_{N} K\right)(\xi, \eta)\right| \leq C\left(C L_{N} /|(\xi,-\eta)|\right)^{(N-M) / 2}, \quad(\xi,-\eta) \in \Gamma_{0},
$$

for some constant $C$ independent of $N$. Since $\chi_{N}=1$ in $B_{r_{0}}\left(x_{0}, y_{0}\right)$, Lemma 1.6 shows that $\left(x_{0}, y_{0}, \xi_{0},-\eta_{0}\right) \notin \mathrm{WF}_{L}(K)$. If $\chi_{N}$ is replaced by

$$
\chi_{0, N}=\overline{\varphi_{N}\left(\cdot-y_{0}\right)} \otimes \varphi_{N}\left(\cdot-x_{0}\right),
$$

then similar arguments show that $\left(y_{0}, x_{0}, \eta_{0},-\xi_{0}\right) \notin \mathrm{WF}_{L}(K)$.

As an immediate consequence of Theorem 2.5 we have the following.

Corollary 2.7. Assume that $K \in \mathscr{D}_{\circ}^{\prime}\left(\mathbb{R}^{2 n}\right) \cap \mathscr{C}^{L}(U)$, where $U \subseteq \mathbb{R}^{2 n}$ is an open neighbourhood of the diagonal $x=y$. Then $K \in \mathscr{C}^{L}\left(\mathbb{R}^{2 n}\right)$.

Next we discuss wave front properties for the Fourier transform of elements in $\mathscr{D}_{0}^{\prime} \cap \mathscr{S}^{\prime}$. 
TheOREM 2.8. Assume that $K \in \mathscr{D}_{\circ}^{\prime}\left(\mathbb{R}^{2 n}\right) \cap \mathscr{S}^{\prime}\left(\mathbb{R}^{2 n}\right)$ and $y, \xi, \eta \in \mathbb{R}^{n}$. If $x \in \mathbb{R}^{n} \backslash 0$ and $(\xi,-\xi, x, x) \notin \mathrm{WF}_{*}(\widehat{K})$, then $(\xi,-\eta, x, y) \notin \mathrm{WF}_{*}(\widehat{K})$ and $(\eta,-\xi, y, x) \notin \mathrm{WF}_{*}(\widehat{K})$, where $\mathrm{WF}_{*}$ stands for $\mathrm{WF}, \mathrm{WF}_{L}$ or $\mathrm{WF}_{\mathcal{A}}$.

Theorem 2.8 is an immediate consequence of Theorem 2.3, Theorem 2.5 and the following result.

Proposition 2.9. Assume that $K \in \mathscr{S}^{\prime}\left(\mathbb{R}^{2 n}\right)$ and let $K_{\mathscr{F}}(\xi, \eta)=$ $\widehat{K}(\xi,-\eta)$. Then $K \in \mathscr{D}_{\circ}^{\prime}\left(\mathbb{R}^{2 n}\right)$ if and only if $K_{\mathscr{F}} \in \mathscr{D}_{\circ}^{\prime}\left(\mathbb{R}^{2 n}\right)$. In particular, if $K \in \mathscr{D}_{\circ}^{\prime}\left(\mathbb{R}^{2 n}\right)$, then (2.2) and the Cauchy-Schwarz inequality

$$
|(\widehat{K}, \varphi \otimes \widetilde{\psi})|^{2} \leq(\widehat{K}, \varphi \otimes \widetilde{\varphi})(\widehat{K}, \psi \otimes \widetilde{\psi})
$$

hold for every $\varphi, \psi \in \mathscr{S}\left(\mathbb{R}^{n}\right)$.

Proof. Assume that $K \in \mathscr{D}_{\circ}^{\prime}$. Then (2.2) holds for all $\varphi, \psi \in C_{0}^{\infty}$, and by a simple approximation argument it also holds for all $\varphi, \psi \in \mathscr{S}$. By Fourier's inversion formula it now follows that

$$
\left(K_{\mathscr{F}}, \varphi \otimes \bar{\varphi}\right)=(\widehat{K}, \varphi \otimes \widetilde{\varphi})=\left(K,\left(\mathscr{F}^{-1} \varphi\right) \otimes \overline{\mathscr{F}-1} \varphi\right) \geq 0 .
$$

This proves that $K_{\mathscr{F}} \in \mathscr{D}_{\circ}^{\prime}$. The result now follows from Fourier's inversion and the obvious fact that $K \in \mathscr{D}_{\circ}^{\prime}$ if and only if $\check{K} \in \mathscr{D}_{\circ}^{\prime}$.

REMARK 2.10. In view of the assumptions in Theorem 2.8 and Proposition 2.9 we note that if $K \in \mathscr{D}_{\circ}^{\prime}\left(\mathbb{R}^{2 n}\right)$ and $K_{\phi}(x, y)=\phi(x-y) K(x, y)$ for some $\phi \in \mathscr{S}\left(\mathbb{R}^{n}\right)$ such that $\phi(0) \neq 0$, then Theorem 2.9 in [16] shows that $K \in \mathscr{S}^{\prime}\left(\mathbb{R}^{2 n}\right)$ if and only if $K_{\phi} \in \mathscr{S}^{\prime}\left(\mathbb{R}^{2 n}\right)$.

REMARK 2.11. Theorems 2.3 and 2.5 do not give any non-trivial information when $K$ is the distribution kernel of a partial differential operator with smooth coefficients.

In fact, any such $K$ can be written as

$$
K(x, y)=\sum_{|\alpha| \leq N} a_{\alpha}(x) \delta_{0}^{(\alpha)}(x-y) .
$$

In particular, $K(x, y)$ is equal to zero, and hence real-analytic, outside $x=y$.

REMARK 2.12. Proposition 2.9 can be considered as a generalization of Bochner's theorem. In fact, assume that $u$ is a positive definite function or distribution, i.e. $K(x, y)=u(x-y)$ belongs to $\mathscr{D}_{\circ}^{\prime} \cap \mathscr{S}^{\prime}$ (see Remark 3.6 in Section 3). Then Proposition 2.9 shows that $K_{\mathscr{F}}(\xi, \eta)=\widehat{u} \otimes \delta(\xi, \eta-\xi)$ belongs to $\mathscr{D}_{\circ}^{\prime}$. This is the same as saying that $\widehat{u}$ is a positive measure.

\section{Wave front set of positive elements in convolution algebras.}

In this section we apply the results from the previous sections to elements in $\mathscr{D}_{B,+}^{\prime}\left(\mathbb{R}^{n}\right)$. To that end we prove that the singularities for such elements are strongest at the origin. 
More precisely, we have the following.

Theorem 3.1. Assume that $B \in C^{*}\left(\mathbb{R}^{2 n}\right), u \in \mathscr{D}_{B,+}^{\prime}\left(\mathbb{R}^{n}\right)$ and $\xi \in \mathbb{R}^{n} \backslash 0$. Then the following hold:

(1) if $(0, \xi) \notin \mathrm{WF}(u)$, then $(x, \xi) \notin \mathrm{WF}(u)$ for every $x \in \mathbb{R}^{n}$;

(2) if $(0, \xi) \notin \mathrm{WF}_{L}(u)$, and in addition $B \in \mathscr{C}^{L}\left(\mathbb{R}^{2 n}\right)$, then $(x, \xi) \notin$ $\mathrm{WF}_{L}(u)$ for every $x \in \mathbb{R}^{n}$;

(3) if $(0, \xi) \notin \mathrm{WF}_{\mathcal{A}}(u)$, and in addition $B \in \mathcal{A}\left(\mathbb{R}^{2 n}\right)$, then $(x, \xi) \notin$ $\mathrm{WF}_{\mathcal{A}}(u)$ for every $x \in \mathbb{R}^{n}$.

Remark 3.2. Assume that $B=1$ everywhere. Then assertion (3) of Theorem 3.1 was proved by Yoshino in [17]. (See also [18].)

The proof of Theorem 3.1 is based on the following lemma.

Lemma 3.3. Assume that $u \in \mathscr{D}^{\prime}\left(\mathbb{R}^{n}\right), f_{1} \in C^{*}\left(\mathbb{R}^{n}\right)$ and $f_{2} \in C^{*}\left(\mathbb{R}^{n}\right) \cap$ $\mathscr{C}^{L}\left(\mathbb{R}^{n}\right)$. Then

$$
\mathrm{WF}\left(f_{1} u\right)=\mathrm{WF}(u), \quad \mathrm{WF}_{L}\left(f_{2} u\right)=\mathrm{WF}_{L}(u),
$$

and

$$
\begin{aligned}
\mathrm{WF}\left(u_{\tau}\right) & =\{(x, y, \xi,-\xi) ;(x-y, \xi) \in \mathrm{WF}(u)\}, \\
\mathrm{WF}_{L}\left(u_{\tau}\right) & =\left\{(x, y, \xi,-\xi) ;(x-y, \xi) \in \mathrm{WF}_{L}(u)\right\} .
\end{aligned}
$$

Proof. The assumptions imply that $1 / f_{1} \in C^{*}$ and $1 / f_{2} \in C^{*} \cap \mathscr{C}^{L}$. Hence (3.1) follows from the inclusions $\operatorname{WF}\left(f_{1} u\right) \subseteq \mathrm{WF}(u)$ and $\mathrm{WF}_{L}\left(f_{2} u\right)$ $\subseteq \mathrm{WF}_{L}(u)$, which are immediate consequences of the definitions and the results in Section 1. (See also [8].)

In order to prove the first equality in $(3.2)$ we let $v(x, y)=(u \otimes 1)(x, y)=$ $u(x)$. In view of Theorem 8.2.4 in [8], the result follows if we prove that

$$
\mathrm{WF}(v)=\{(x, y, \xi, 0) ;(x, \xi) \in \mathrm{WF}(u)\} .
$$

Let $\chi(x, y)=\chi_{1}(x) \chi_{2}(y)$, where $\chi_{1}, \chi_{2} \in C_{0}^{\infty}\left(\mathbb{R}^{n}\right)$. Then

$$
\mathscr{F}(\chi v)(\xi, \eta)=\mathscr{F}\left(\chi_{1} u\right)(\xi) \widehat{\chi}_{2}(\eta) .
$$

Since $\widehat{\chi}_{2}$ is rapidly decreasing, and $\left|\mathscr{F}\left(\chi_{1} u\right)(\xi)\right| \leq C\langle\xi\rangle^{M}$ when $\xi \in \mathbb{R}^{n}$, for some constants $C$ and $M$, it follows that $(\xi, \eta) \in \Sigma(\chi v)$ if and only if $\xi \in \Sigma(u)$ and $\eta=0$. This proves the first equality in (3.2).

The second equality follows by similar arguments, using Theorem 8.5.1 instead of Theorem 8.2.4 in [8].

Proof of Theorem 3.1. (1) Let $K=u_{\tau} B$, and assume that $(0, \xi) \notin$ $\mathrm{WF}(u)$. Then it follows from Lemma 3.3 that

$$
(x, x, \xi,-\xi) \notin \mathrm{WF}\left(u_{\tau}\right)=\mathrm{WF}(K) .
$$

Since we have $K \in \mathscr{D}_{\circ}^{\prime}$, Theorem 2.3 implies that $(x, 0, \xi,-\xi) \notin \mathrm{WF}(K)$, i.e. $(x, 0, \xi,-\xi) \notin \mathrm{WF}\left(u_{\tau}\right)$. Hence $(3.2)$ shows that $(x, \xi) \notin \mathrm{WF}(u)$. 
Assertions (2) and (3) follow by similar arguments, using Theorem 2.5 instead of Theorem 2.3. The details are left for the reader.

Next we list some consequences of Theorem 3.1. The first result concerns smoothness near the origin. (See $[4,12,17]$ or $[18]$ in the case of standard convolution, i.e. the case when $B=1$.)

Corollary 3.4. Assume that $B \in C^{*}\left(\mathbb{R}^{2 n}\right), u \in \mathscr{D}_{B,+}^{\prime}\left(\mathbb{R}^{n}\right)$, and $U \subseteq$ $\mathbb{R}^{n}$ is an open neighbourhood of origin. Then the following holds:

(1) if in addition $u \in C^{\infty}(U)$, then $u \in C^{\infty}\left(\mathbb{R}^{n}\right)$;

(2) if in addition $B \in \mathscr{C}^{L}\left(\mathbb{R}^{2 n}\right)$ and $u \in \mathscr{C}^{L}(U)$, then $u \in \mathscr{C}^{L}\left(\mathbb{R}^{n}\right)$;

(3) if in addition $B \in \mathcal{A}\left(\mathbb{R}^{2 n}\right)$ and $u \in \mathcal{A}(U)$, then $u \in \mathcal{A}\left(\mathbb{R}^{n}\right)$.

By combining Theorem 2.3 with Theorem 3.1, we are now able to prove the following generalization of Proposition 2.1.

Proposition 2.1'. Assume that $K \in \mathscr{D}_{\circ}^{\prime}\left(\mathbb{R}^{2 n}\right)$ and $f \in \mathscr{D}_{1,+}^{\prime}\left(\mathbb{R}^{n}\right)$ are such that

$$
\{(x, x, \xi,-\xi) \in \mathrm{WF}(K) ;(0,-\xi) \in \mathrm{WF}(f)\}=\emptyset .
$$

Then $f_{\tau} K$ is uniquely determined as an element in $\mathscr{D}^{\prime}\left(\mathbb{R}^{2 n}\right)$ by the pullback of the tensor product $f_{\tau} \otimes K$ by the diagonal map $(x, y) \mapsto(x, y, x, y)$. Furthermore, $f_{\tau} K \in \mathscr{D}_{\circ}^{\prime}\left(\mathbb{R}^{2 n}\right)$.

Proof. By Theorems 2.3 and 3.1 it follows that

$$
\left\{(x, y, \xi,-\eta) \in \mathrm{WF}(K) ;(x, y,-\xi, \eta) \in \mathrm{WF}\left(f_{\tau}\right)\right\}=\emptyset .
$$

Hence $K_{1}=f_{\tau} K$ is well-defined in view of Theorem 8.2.10 in [8]. This proves the first part of the proposition.

It remains to prove that $f_{\tau} K \in \mathscr{D}_{\circ}^{\prime}$. Let $\varepsilon_{j}>0$ be a sequence which decreases to zero as $j$ goes to infinity, and set $\chi_{j}=\varepsilon_{j}^{-n} \chi\left(\cdot / \varepsilon_{j}\right)$, where $\chi \in C_{0}^{\infty}\left(\mathbb{R}^{n}\right)$ is chosen such that $\int \chi d x=1$ and $\hat{\chi}$ is non-negative. Also let $f_{j}=f * \chi_{j}$ and $f_{j, \tau}=\left(f_{j}\right)_{\tau}$. If the pullback and the distribution in Theorem 8.2.4 in [8] are chosen as $(x, y) \mapsto(x, y, x, y)$ and $f_{j, \tau} \otimes K$ respectively, then $f_{j, \tau} K \rightarrow f_{\tau} K$ in $\mathscr{D}^{\prime}$ as $j \rightarrow \infty$. Since $\widehat{f}_{j}=(2 \pi)^{n / 2} \widehat{\chi}_{j} \widehat{f}$ is a non-negative measure, it follows that $f_{j} \in \mathscr{D}_{1,+}^{\prime} \cap C^{\infty}$. Hence Proposition 2.1 shows that $f_{j, \tau} K \in \mathscr{D}_{\mathrm{O}}^{\prime}$. This gives

$$
\left(f_{\tau} K, \varphi \otimes \bar{\varphi}\right)=\lim _{j \rightarrow \infty}\left(f_{j, \tau} K, \varphi \otimes \bar{\varphi}\right) \geq 0
$$

when $\varphi \in C_{0}^{\infty}$. Hence $f_{\tau} K \in \mathscr{D}_{\circ}^{\prime}$, and the proof is complete.

Corollary 3.5. Assume that $u \in \mathscr{D}_{B,+}^{\prime}\left(\mathbb{R}^{n}\right)$ and $f \in \mathscr{D}_{1,+}^{\prime}\left(\mathbb{R}^{n}\right)$ are such that

$$
\{(0, \xi) \in \mathrm{WF}(u) ;(0,-\xi) \in \mathrm{WF}(f)\}=\emptyset .
$$


Then $f u$ is uniquely determined as the pullback of the tensor product $f \otimes u$ by the diagonal map $x \mapsto(x, x)$. Furthermore $f u \in \mathscr{D}_{B,+}^{\prime}\left(\mathbb{R}^{n}\right)$.

Proof. The result follows immediately from Lemma 3.3, Proposition 2.1' and the fact that $f u \in \mathscr{D}_{B,+}^{\prime}$ if and only if $f_{\tau} u_{\tau} B \in \mathscr{D}_{\circ}^{\prime}$.

REMARK 3.6. Some other properties for $\mathscr{D}_{B,+}^{\prime}$ can be found in [16] when $B \in C^{*}\left(\mathbb{R}^{2 n}\right)$. For example, let $\mathscr{S}_{B,+}^{\prime}\left(\mathbb{R}^{n}\right)=\mathscr{D}_{B,+}^{\prime}\left(\mathbb{R}^{n}\right) \cap \mathscr{S}^{\prime}\left(\mathbb{R}^{n}\right)$. Also assume that for each multi-index $\alpha$, there is a polynomial $P_{\alpha}$ such that (0.3) holds. Then it is proved in Section 2 of [16] that $\mathscr{D}_{B,+}^{\prime}\left(\mathbb{R}^{n}\right)=\mathscr{S}_{B,+}^{\prime}\left(\mathbb{R}^{n}\right)$. In particular, if $B=1$, then "the classical" Bochner-Schwartz theorem follows.

Here, as in the proof of Theorem 2.3, an important idea for the proof is that if $u \in \mathscr{D}_{B,+}^{\prime}$, then the map $(\varphi, \psi) \mapsto\left(u *_{B} \varphi, \psi\right)$ defines a semi-scalar product on $C_{0}^{\infty}\left(\mathbb{R}^{n}\right)$, which in particular implies that the Cauchy-Schwarz inequality holds, i.e.

$$
\left|\left(u *_{B} \varphi, \psi\right)\right|^{2} \leq\left(u *_{B} \varphi, \varphi\right)\left(u *_{B} \psi, \psi\right) .
$$

REMARK 3.7. There are several extensions of the Bochner-Schwartz theorem concerning the standard convolution (i.e. the case $B=1$ in Remark 3.6 ), especially in the theory of hyperfunctions. (See e.g. $[3-5,10,12,13$, $17,18]$.)

\section{Applications to positive elements in the twisted convolution}

algebra. In this section we apply the results of the previous section to the twisted convolution algebra. (Cf. Section 1.) From the definition it follows that the twisted convolution is equal to $*_{B}$ with $B$ as in (0.4), which is nonzero and real-analytic everywhere. Consequently, all positivity results in the previous sections are valid for the twisted convolution. However, by taking into account the special structure of the twisted convolution it turns out that regularity at the origin for positive elements does not only impose similar regularity for such elements globally, but also for the Fourier transform of such elements. Such questions were carefully investigated in [16], and the considerations in the present section can be viewed as a continuation of that discussion.

For example, the WF case in the following theorem is a restatement of Theorem 4.14 in [16]. Here recall from the introduction that $\mathscr{S}_{+}^{\prime}\left(\mathbb{R}^{2 n}\right)=$ $\mathscr{D}_{B,+}^{\prime}\left(\mathbb{R}^{2 n}\right)$ when $*_{B}$ is the twisted convolution. (Note that this implies that $\mathscr{S}_{+}^{\prime} \subseteq \mathscr{S}^{\prime}$ in view of Remark 3.6.)

TheOREM 4.1. Assume that $u \in \mathscr{S}_{+}^{\prime}\left(\mathbb{R}^{2 n}\right)$ and that $Y \in \mathbb{R}^{2 n} \backslash 0$. If $(0, Y) \notin \mathrm{WF}_{*}(u)$ and $X \in \mathbb{R}^{2 n}$, then $(X, Y) \notin \mathrm{WF}_{*}(u)$ and $(X, Y) \notin$ $\mathrm{WF}_{*}\left(\mathscr{F}_{\sigma} u\right)$, where $\mathrm{WF}_{*}$ stands for $\mathrm{WF}, \mathrm{WF}_{L}$ or $\mathrm{WF}_{\mathcal{A}}$. 
Proof. We have to prove the assertion for $\mathrm{WF}_{L}$ and $\mathrm{WF}_{\mathcal{A}}$. Since $B(X, Y)$ $=e^{2 i \sigma(X, Y)}$ is real-analytic, the latter assertion is a consequence of the former.

In order to prove the assertion for $\mathrm{WF}_{L}$ we observe that the assertion is invariant under the choice of Fourier transform. Hence we may assume that the symplectic Fourier transform has been used in the definition of wave front set. Assume that $\left(0, Y_{0}\right) \notin \mathrm{WF}_{L}(u)$, and that $X_{0} \in \mathbb{R}^{2 n}$ is arbitrary. Then $\left(X_{0}, Y_{0}\right) \notin \mathrm{WF}_{L}(u)$ in view of Theorem 3.1, and from the definitions and Lemma 1.5 there is an open neighbourhood $U$ of the origin and a conical neighbourhood $\Gamma$ of $Y_{0}$ such that

$$
\left|\mathscr{F}_{\sigma}\left(u \phi_{N}\right)(Y)\right| \leq C\left(C L_{N} /|Y|\right)^{N}, \quad\left\{\phi_{N}\right\} \in \Lambda^{L}(U),
$$

where the constant $C$ is independent of $N$.

Next choose $r>0$ such that $B_{2 r}(0) \subseteq U$ and

$$
\begin{aligned}
& \left|\arg \left(e^{2 i \sigma\left(X_{1}, Y_{1}\right)}\right)-\arg \left(e^{2 i \sigma\left(X_{2}, Y_{2}\right)}\right)\right| \leq \pi / 8 \text { when } \\
& X_{1}, X_{2} \in B_{2 r}\left(X_{0}\right), Y_{1}, Y_{2} \in B_{2 r}(0) .
\end{aligned}
$$

Also let $\left\{\varphi_{N}\right\} \in \Lambda^{L}\left(B_{r}(0)\right)$ and $\left\{\psi_{N}\right\} \in \Lambda^{L}\left(B_{r}\left(X_{0}\right)\right.$ be sequences of nonnegative functions such that $\varphi_{N}(X)=\psi_{N}\left(X+X_{0}\right)=1$ for every $N$ and $X \in B_{r / 2}(0)$. Finally, let $\phi_{N}=\check{\psi}_{N}$, and

$$
\begin{aligned}
& \varphi_{N, Y}=e^{-2 i \sigma(Y, \cdot)} \varphi_{N}, \quad \psi_{N, Y}=e^{2 i \sigma(Y, \cdot)} \psi_{N}, \quad \phi_{N, Y}=\check{\psi}_{N, Y}, \\
& \bar{\chi}_{N}=\psi_{N} *_{\sigma} \widetilde{\varphi}_{N}, \quad \bar{\chi}_{1, N}=\varphi_{N} *_{\sigma} \widetilde{\varphi}_{N}, \quad \bar{\chi}_{2, N}=\phi_{N} *_{\sigma} \widetilde{\phi}_{N} \text {. }
\end{aligned}
$$

Then $\left\{\chi_{N}\right\} \in \Lambda^{L}\left(B_{2 r}\left(X_{0}\right)\right)$ and $\left\{\chi_{j, N}\right\} \in \Lambda^{L}\left(B_{2 r}(0)\right)$ for $j=1,2$, and by (4.1) there exists a constant $c>0$ such that

$$
\left|\chi_{N}\left(X+X_{0}\right)\right| \geq c, \quad\left|\chi_{1, N}(X)\right| \geq c, \quad\left|\chi_{2, N}(X)\right| \geq c
$$

for every $N$ and $X \in B_{r}(0)$.

By Proposition 1.8, (3.5) and straightforward computations we get

$$
\begin{aligned}
\left|\mathscr{F}_{\sigma}\left(\widehat{u} \chi_{N}\right)(Y)\right|^{2} & =\left|\left(e^{2 i \sigma(Y, \cdot)} \widehat{u}, \psi_{N} *_{\sigma} \widetilde{\varphi}_{N}\right)\right|^{2} \\
& =\left|\left(\widehat{u}, \psi_{N, Y} *_{\sigma} \widetilde{\varphi}_{N, Y}\right)\right|^{2}=\left|\left(u, \widehat{\psi}_{N, Y} *_{\sigma} \widetilde{\varphi}_{N, Y}\right)\right|^{2} \\
& \leq\left(u, \varphi_{N, Y} *_{\sigma} \widetilde{\varphi}_{N, Y}\right)\left(u, \mathscr{F}_{\sigma} \psi_{N, Y} *_{\sigma} \widetilde{\mathscr{F}}_{\sigma} \psi_{N, Y}\right) \\
& =\left(u, \varphi_{N, Y} *_{\sigma} \widetilde{\varphi}_{N, Y}\right)\left(u, \phi_{N, Y} *_{\sigma} \widetilde{\phi}_{N, Y}\right) .
\end{aligned}
$$

Since

$$
\begin{aligned}
\varphi_{N, Y} *_{\sigma} \widetilde{\varphi}_{N, Y} & =e^{-2 i \sigma(Y, \cdot)} \varphi_{N} *_{\sigma} \widetilde{\varphi}_{N}=e^{-2 i \sigma(Y, \cdot)} \bar{\chi}_{1, N}, \\
\phi_{N, Y} *_{\sigma} \widetilde{\phi}_{N, Y} & =e^{-2 i \sigma(Y, \cdot)} \phi_{N} *_{\sigma} \widetilde{\phi}_{N}=e^{-2 i \sigma(Y, \cdot)} \bar{\chi}_{2, N},
\end{aligned}
$$

by straightforward computations, it follows that

$$
\left(u, \varphi_{N, Y} *_{\sigma} \widetilde{\varphi}_{N, Y}\right)=\mathscr{F}_{\sigma}\left(\chi_{1, N} u\right)(Y), \quad\left(u, \phi_{N, Y} *_{\sigma} \widetilde{\phi}_{N, Y}\right)=\mathscr{F}_{\sigma}\left(\chi_{2, N} u\right)(Y) .
$$


From the above estimates we get

$$
\left|\mathscr{F}_{\sigma}\left(\chi_{N} \widehat{u}\right)(Y)\right|^{2} \leq \mathscr{F}_{\sigma}\left(\chi_{1, N} u\right)(Y) \mathscr{F}_{\sigma}\left(\chi_{2, N} u\right)(Y)
$$

It now follows that (1.4) holds with $u_{N}=\chi_{N} \widehat{u}$. Hence $\left(X_{0}, Y_{0}\right) \notin \mathrm{WF}_{L}(\widehat{u})$, and the assertion follows.

As a consequence of Theorem 4.1, if $u \in \mathscr{S}_{+}^{\prime}\left(\mathbb{R}^{2 n}\right) \cap C^{\infty}(U)$, then $u$ and $\widehat{u}$ belong to $C^{\infty}\left(\mathbb{R}^{2 n}\right)$. A stronger result in this context is $(1)$ in the following refinement of Corollary 3.4 in the case of twisted convolution.

THEOREM 4.2. Assume that $U \subseteq \mathbb{R}^{2 n}$ is an open neighbourhood of the origin. Then the following hold:

(1) if $u \in \mathscr{S}_{+}^{\prime}\left(\mathbb{R}^{2 n}\right) \cap C^{\infty}(U)$, then $u \in \mathscr{S}\left(\mathbb{R}^{2 n}\right)$;

(2) if $u \in \mathscr{S}_{+}^{\prime}\left(\mathbb{R}^{2 n}\right) \cap \mathscr{C}^{L}(U)$, then $u$ and $\widehat{u}$ belong to $\mathscr{C}^{L}\left(\mathbb{R}^{2 n}\right) \cap \mathscr{S}\left(\mathbb{R}^{2 n}\right)$;

(3) if $u \in \mathscr{S}_{+}^{\prime}\left(\mathbb{R}^{2 n}\right) \cap \mathcal{A}(U)$, then $u$ and $\widehat{u}$ belong to $\mathcal{A}\left(\mathbb{R}^{2 n}\right) \cap \mathscr{S}\left(\mathbb{R}^{2 n}\right)$.

Proof. Assertion (1) follows immediately from Theorem 3.13 in [16]. The rest is an immediate consequence of Theorem 4.1 and (1).

REMARK 4.3. Assume that $K(X-Y)=u(X-Y) e^{2 i \sigma X, Y)}$ belongs to $\mathscr{D}_{\mathrm{o}}^{\prime}$, i.e. $u \in \mathscr{S}_{+}^{\prime}$. Then the Fourier inversion formula gives that $\mathscr{F}_{\sigma} K(X,-Y)=$ $u(Y-X) e^{2 i \sigma X, Y)}$. (See the introduction and Remark 3.6.) Hence, Proposition 2.9 asserts that $u \in \mathscr{S}_{+}^{\prime}$ if and only if $\check{u} \in \mathscr{S}_{+}^{\prime}$. On the other hand, the last property follows immediately from the definitions, which in particular implies that Proposition 2.9 does not give any non-trivial information for elements in $\mathscr{S}_{+}^{\prime}$. Consequently, Theorem 2.8 does not give any further information compared to Theorems 2.3 and 2.5 in the case of twisted convolution.

REMARK 4.4. A question which arises here is whether the condition $\mathrm{WF}(u)=\mathrm{WF}(\widehat{u})=\emptyset$ (i.e. $u, \widehat{u} \in C^{\infty}$ ) for a distribution $u$ implies that indeed $u \in \mathscr{S}$. The answer is negative. In fact, if $u(x)=e^{i|x|^{2}}$, then $u, \widehat{u} \in$ $C^{\infty} \cap \mathscr{S}^{\prime} \backslash \mathscr{S}$. We refer to [6] for a brief investigation of such questions.

Acknowledgements. I am grateful to Professors D. Perišić and L. Rodino for fruitful discussions and valuable advice.

\section{References}

[1] S. Bochner, Vorlesungen über Fouriersche Integrale, Akademie-Verlag, Leipzig, 1932.

[2] M. Budinčević, Z. Lozanov-Crvenković and D. Perišić, Representation theorems for tempered ultradistributions, Publ. Inst. Math. (Beograd) (N.S.) 65 (79) (1999), 142160.

[3] J. Chung, S. Y. Chung and D. Kim, Positive definite hyperfunctions, Nagoya J. Math. 140 (1995), 139-149. 
[4] S. Y. Chung, Quasi analyticity of positive definite continuous functions, Publ. RIMS Kyoto Univ. 38 (2002), 725-733.

[5] S. Y. Chung and K. Dohan, Distributions with exponential growth and BochnerSchwartz theorem for Fourier hyperfunctions, ibid. 31 (1995), 829-845.

[6] S. Coriasco and L. Maniccia, Wave front set at infinity and hyperbolic linear operators with multiple characteristics, Ann. Global Anal. Geom. (4) 24 (2003), 375-400.

[7] G. B. Folland, Harmonic Analysis in Phase Space, Princeton Univ. Press, Princeton, 1989.

[8] L. Hörmander, The Analysis of Linear Partial Differential Operators, Vols. I, III, Springer, Berlin, 1983, 1985.

[9] H. Komatsu, Microlocal analysis in Gevrey classes and in complex domains, in: L. Cattabriga and L. Rodino (eds.), Microlocal Analysis and Applications, Springer, Berlin, 1991, 161-236.

[10] Z. Lozanov-Crvenković and D. Perišić, Generalized Bochner-Schwartz theorem for tempered ultradistributions, Novi Sad J. Math. 29 (1999), 69-82.

[11] M. Reed and B. Simon, Methods of Modern Mathematical Physics, Academic Press, London, 1979.

[12] M. Suwa, Fourier ultra-hyperfunctions as boundary values of smooth solutions of the heat equation, Tokyo J. Math. 25 (2002), 381-398.

[13] M. Suwa and K. Yoshino, A characterization of tempered distributions with support in a cone by the heat kernel method and its applications, J. Math. Sci. Univ. Tokyo 11 (2004), 75-90.

[14] J. Toft, Continuity and positivity problems in pseudo-differential calculus, Thesis, Dept. Math., Univ. of Lund, Lund, 1996.

[15] - Continuity properties for non-commutative convolution algebras with applications in pseudo-differential calculus, Bull. Sci. Math. (2) 126 (2002), 115-142.

[16] - Positivity properties for non-commutative convolution algebras with applications in pseudo-differential calculus, ibid. 127 (2003), 101-132.

[17] K. Yoshino, Propagation of micro analyticity of positive definite distributions, preprint, 2005.

[18] - Recent development of heat kernel method in the theory of generalized functions, preprint, 2006.

Department of Mathematics and Systems Engineering

Växjö University

S-351 95 Växjö, Sweden

E-mail: joachim.toft@vxu.se

Received April 10, 2006

Revised version November 3, 2006 